$0.1312 \mathrm{~g}$ Sbst.: $0.3587 \mathrm{~g} \mathrm{CO}_{2}, 0.0788 \mathrm{~g} \mathrm{H}_{2} \mathrm{O} .-0.136 \mathrm{~g}$ Sbst.: $03717 \mathrm{~g}$ $\mathrm{CO}_{2}, 0.0826 \mathrm{~g} \mathrm{H}_{2}$ 0. $-0.1068 \mathrm{~g}$ Sbst.: $18.25 \mathrm{ccm} \mathrm{N}(210,739 \mathrm{~mm})$.

$$
\begin{aligned}
& \mathrm{C}_{14} \mathrm{H}_{15} \mathrm{~N}_{3} \text {. Ber. C 74.68, H 6.66, N 18.66. } \\
& \text { Gef. \ 74.56, 74.54, » 6.67, 6.74, 》 } 18.90 \text {. }
\end{aligned}
$$

$0.1925 \mathrm{~g}$ Sbst. in $20.3 \mathrm{~g}$ Benzol gaben eine Gefrierpunktserniedrigung von 0.1980. - 0.3272 g Sbst. in $20.3 \mathrm{~g}$ Benzol gaben eine Gefrierpunktserniedrigung von $0.838^{\circ}$.

$$
\text { Mol.-Gew. Ber. 225. Gef. 239, } 239 .
$$

Aufspaltung mit Alkali.

Dieselbe erfolgt durch Erhitzen mit verdünntem Alkali im Robr bei $150^{\circ}$ noch nicht, $0.3 \mathrm{~g}$ Base wurden mit festem Aetzkali im Reagensglase rückfliessend erhitzt, bis die gelbe Farbe des Oeles verschwunden war. Die Schmelze wurde dann in Wasser gelöst und mit Aether extrahirt; nach dem Verdunsten des Lösungsmittels blieben, neben anderen, nicht näher charakterisirbaren Substanzen, Spuren von Methylanilin zurück. Die alkalische Lösung, mit verdünnter Salzsäare angesäuert, schied farblose Krystalle ab, welche sich als Methylanthranilsäure erwiesen and mit einem Vergleichspräparat nach Schmelzpunkt, Krystallform und Eigenschaften übereinstimmten. Ausbeute $0.06 \mathrm{~g}$ reine, aus Ligroïn umkrystallisirte Säure.

703. August Klages und S. Margolinsky: Synthese von Betaïnen aus dialkylirten Aminonitrilen.

(Fingegangen am 2. December 1903.)

Dialkylirte Aminonitrile, $\mathrm{R}_{2} \mathrm{~N} . \mathrm{CH}_{2} . \mathrm{CN}$, vereinigen sich mit Jodalkylen zu jodwasserstoffsauren Salzen $R_{3} N(J) \cdot C_{2} \cdot C N$, die mit feucbtem Silberoxyd quaternäre Basen ${ }^{2}$ ) liefern. Ich babe sie s. Z. als Nitrile der Betaine $\mathrm{R}_{3} \mathrm{~N}(\mathrm{OH}) \cdot \mathrm{CH}_{2}$. $\mathrm{CN}$ bezeichnet. Diesen Namen verdienen die Basen mit Recht, denn sie gehen durch Verseifung unter Verlust von Ammoniak in die Betaine selbst über.

Die Nitrile der Betaïne sind stark alkalisch reagirende Syrupe. Sie haben die Fähigkeit, Silber in Lösung zu halten, und sind deshalb schwer zu reinigen. Entfernt man das Silber vorsichtig durch Salzsäure, so erbält man Basen, die durch ibr Chlorid verunreinigt sind; wendet man Kohlensäure zur Fällung des Silbers an, so entstehen, neben unlöslichem kohlensaurem Silber, Carbonate, die sich durch Eindampfen der Lösung im Vacuum gewinnen lassen. Aus diesen

ग) A. Klages, Journ. für prakt. Chem. [2] 65, 188 [1902]. 
Carbonaten kann durch Digeriren mit Zinkhydroxyd die Kohlensäure nicht entfernt werden. Dampft man die silberbaltigen Lösungen direct ein, so scheidet sich das Silter theils als Spiegel, theils als graues Pulver ab. Die erhaltenen silberfreien Syrupe zeigen jedoch nicht den richtigen Stickstoffgehalt. Sie sind Geonische von Nitrilen und den durch Verseifnng daraus entstandenen Betaïnen. Wenn man reine Derivate der Nitrile herstellen will, ist es daher nöthig, die mit Silberhydroxyd in der Kälte bereiteten Lösungen der Basen zu benutzen. Es entstehen mit wässrigen Lösungen von Pikrinsäure gut krystallisirende Pikrate, Doppelverbindungen, die aus $1 \mathrm{Mol}$. Pikrinsäure und $1 \mathrm{Mol}$. des Nitrils zusammengesetzt sind. Goldchlorid liefert mit salzsauren Lösungen die entsprechenden goldchlorwasserstoffsauren Salze, Sublimat bisweilen complicirte Salze der Formel $\mathbf{R}_{3} \mathrm{~N}(\mathrm{Cl}) \cdot \mathrm{CH}_{2} \cdot \mathrm{CN} . \mathrm{HgCl}+\mathrm{Hg} \mathrm{Cl}_{2}$.

Beim Kochen mit Barythydrat entstehen aus den Nitrilen die zugehörigen Betaïne, die leicht auf diese Weise zu gewinnen sind. Man fällt den Baryt heiss durch Kohlensäure und dampft die filtriı ten Lösungen zur Trockne. Den Rückstand nimmt man in absolutem Alkohol auf, filtrirt ab und engt die Lösung auf dem Wasserbade ein. Es krystallisiren die Betaïne dann beim Verdunsten des Alkohols meist in radialstrahligen Rosetten aus, die über Schwefelsäure allmählich zu einem weissen Krystallpulver rerwittern. Das völlige Trocknen der sehr bygroskopischen Betaine erfordert oft wochenlanges Evacuiren über Schwefelsäure, wie das uuch Willstätter bei seinen Untersuchungen beobachtet hat.

\section{Metbyl-diäthyl-betaĩn, $\left(\mathrm{CH}_{3}\right)\left(\mathrm{C}_{2} \mathrm{H}_{5}\right)_{2} \mathrm{~N} . \mathrm{CH}_{2}$. CO. O.}

Das Diäthylamino-acetonitril, das ich durch Erhitzen von Glykolsäurenitril mit Diäthylamin dargestellt habe, lässt sich noch einfacher gewinnen, wenn man $15 \mathrm{~g} \mathrm{40-proc.} \mathrm{Formaldehyd} \mathrm{mit} 55 \mathrm{~g}$ Bisulfitlauge mischt, das in Lösung befindliche oxymethylensulfosaure Natrium mit $20 \mathrm{~g}$ Cyankalium in $40 \mathrm{~g}$ Wasser umsetzt und $12 \mathrm{~g}$ Diäthylamin hinzufügt. Es scheidet sich das Nitril dann sofort als Oelschicht aus.

Das Jodmethylat des Diäthylamino-acetonitrils schmilat bei $190-191^{\circ}$ (nicht, wie früher angegeben, bei $205^{\circ}$ ). Es krystallisirt aus Alkohol in breiten Blättern.

Mit feucbtem Silberoxyd entsteht die stark alkalisch reagirende Lösung der freien Base, die mit wässriger Pikrinsäurelösung eine reichliche Fällung des Pikrates liefert.

Das Pikrat des Methyl-diäthyl-betaïnitrils bildet feine, gelbe Nadeln, die aus Wasser ausgezeichnet krystallisiren. Schmp. 166 $-168^{\circ}$ obne Zersetzung. 
$0.1040 \mathrm{~g}$ Sbst.: $0.1860 \mathrm{~g} \mathrm{CO}_{2}, 0.0385 \mathrm{~g} \mathrm{H}_{2} \mathrm{O} .-0.1323 \mathrm{~g}$ Sbst.: $22.5 \mathrm{ccm}$ N $\left(24^{0}, 752 \mathrm{~mm}\right)$.

$\mathrm{C}_{7} \mathrm{H}_{16} \mathrm{~N}_{2} \mathrm{O}+\mathrm{C}_{6} \mathrm{H}_{2}\left(\mathrm{NO}_{2}\right)_{3} . \mathrm{OH}$.

Ber. C 48.8 , H $4.1, \mathrm{~N} 18.76$.

Gef. 》 48.8 , 》 4.1 , 》 18.86 .

Das durch Verseifen mit Barytwasser dargestellte Methyldiätbyl-betaïn zeigte die von Willstätter ${ }^{1}$ ) angegebenen Eigenschaften. Das Chlorid des Betains krystallisirt aus wenig heissem Wasser in glänzenden Blättchen. Schmp. $194^{\circ}$.

0.1502 g Sbst.: $10 \mathrm{~cm} \mathrm{~N}\left(20^{\circ}, 759 \mathrm{~mm}\right)$.

$$
\mathrm{C}_{7} \mathrm{H}_{16} \mathrm{O}_{2} \mathrm{NCl} \text {. Ber. } \mathrm{N} \text { 7.7. Gef. N 7.6. }
$$

Das Pikrat des Betains krystallisirt in derben, gut ausgebildeten, glänzenden Nadeln. Es scbmilzt bei $153-154^{0}$.

$0.1644 \mathrm{~g}$ Sbst.: $0.2543 \mathrm{~g} \mathrm{CO}_{2}, 0.0706 \mathrm{~g} \mathrm{H}_{2} \mathrm{O} .-0.0767 \mathrm{~g}$ Sbst.: $0.1176 \mathrm{~g}$ $\mathrm{CO}_{2}, 0.0394 \mathrm{~g} \mathrm{H}_{2} \mathrm{O}$.

$$
\begin{array}{ll}
\mathrm{C}_{17} \mathrm{H}_{15} \mathrm{NO}_{2}+\mathrm{C}_{6} \mathrm{H}_{2}\left(\mathrm{NO}_{2}\right)_{3} \mathrm{OH} . & \begin{array}{l}
\text { Ber. C } 42.1, \\
\text { Gef. }
\end{array} \\
& \text { H } 4.7,41.8 \text {, 》 } 4.8,5.0 .
\end{array}
$$

\section{Triäthyl-betain, $\left(\mathrm{C}_{2} \mathrm{H}_{6}\right)_{3} \mathrm{~N} \cdot \mathrm{CH}_{2} \cdot \mathrm{CO} \cdot \mathrm{O}$.}

Für das Jodäthylat des Diäthylamino-acetonitril batte ich einen Schmelzpunkt von $179^{\circ}$ angegeben. Durch wiederholtes Umkrystallisiren der Substanz aus Alkohol stieg der Schmelzpunkt bis auf $184^{\circ}$. Ich halte diesen Schmelzpunkt für den richtigen.

Die mit feuchtem Silberoxyd in der Kälte bereitete Lösung des Triätbylbetaïnnitrils lieferte ein aus beissem Wasser in glänzenden Nadeln krystallisirendes Pikrat. Schmp. $193^{\circ}$.

$0.249 \mathrm{~g}$ Sbst.: $39.8 \mathrm{ccm} \mathrm{N}\left(22^{n}, 742 \mathrm{~mm}\right)$.

$\mathrm{C}_{14} \mathrm{H}_{21} \mathrm{~N}_{5} \mathrm{O}_{8}$. Ber. N 18.09. Gef. N 17.66 .

Das goldchlorwasserstoffsaure Salz fällt als gelber Niederschlag beim Versetzen der salzsauren Lösung des Nitrils mit 10-proc. Goldcbloridlösung aus. Schmp. 118-1190.

$0.142 \mathrm{~g}$ Sbst.: $0.0582 \mathrm{~g} \mathrm{Au}$.

$\mathrm{C}_{8} \mathrm{H}_{12} \mathrm{~N}_{2} \mathrm{Cl}_{4} \mathrm{Au}$. Ber. An 41.04. Gef. Au 40.99 .

Mit einer kaltgesättigten Lösung ron Sublimat entstebt eine weisse Fällung des Quecksilberdoppelsalzes, welches aus Alkohol sich in breiten, glänzenden Blättern abscheidet. Es schmilzt bei 130-131". $\mathrm{AgCl}$.

$0.1708 \mathrm{~g}$ Sbst.: $5.8 \mathrm{ccm} \mathrm{N}\left(14^{0}, 742 \mathrm{~mm}\right)$ - $-0.1444 \mathrm{~g}$ Sbst.: $0.136 \mathrm{~g}$ $\mathrm{C}_{8} \mathrm{H}_{17} \mathrm{~N}_{2} \mathrm{Hg}_{2} \mathrm{Cl}_{4}$. Ber. N 3.9, Cl 23.34. Gef. » $3.9, » 23.28$. 
Beim Kochen mit der dreifachen Menge Barythydrat geht das Nitril unter Abspaltung von Ammoniak in Triäthyl-betaïn ${ }^{1}$ ) über. Der ausführlichen Beschreibung Willstätter's habe ich noch hinzuzufügen, dass das Aurochlorat aus heissem Wasser in Nadeln krystallisirt und bei $162^{0}$ schmilzt.

$0.1139 \mathrm{~g}$ Sbst.: $0.0449 \mathrm{~g}$ Au.

$\mathrm{C}_{8} \mathrm{H}_{17} \mathrm{NO}_{2} \mathrm{AuCl}_{4}$. Ber. Au 39.96. Gef. Au 39.42.

a- Iethyl-diäi hyl-propiobetän' $\left.{ }^{2}\right),\left(\mathrm{CH}_{3}\right)\left(\mathrm{C}_{2} \mathrm{H}_{5}\right)_{2} \mathrm{~N} \cdot \mathrm{CH}\left(\mathrm{CH}_{3}\right) \cdot \mathrm{CO} . \mathrm{O}$.

Das Jodmethylat des $\alpha$-Diäthylamino-propionitrils wurde durch Einwirkung von Jodmethyl auf das Nitril in d er Kält e erbalten. Nach 24 Stunden war die Lösung zu einem farblosen Krystallbrei erstarrt. Schmp. 195-196 ${ }^{\circ}$ unter Zersetzung. Durch Behandeln mit Silberbydroxyd und Kochen der erbaltenen Lösung des Nitrils mit Barythydrat entsteht das Methyl-diäthyl-propiobetaĩn. Es bildet eine langstrahlige Krystallmasse, die über Schwefelsäure verwittert und schliesslich zu einem weissen Pulrer zerfällt, das von $117-119^{\circ}$ unter vorherigem Erweichen schmilzt.

$0.1470 \mathrm{~g}$ Sbst.: $0.3234 \mathrm{~g} \mathrm{CO}_{2}, 0.1460 \mathrm{~g} \mathrm{H}_{2} \mathrm{O} .-0.1722 \mathrm{~g}$ Sbst.: $13.5 \mathrm{ccm}$ $\mathrm{N}\left(21^{0}, 7.53 \mathrm{~mm}\right)$.

$$
\begin{aligned}
& \mathrm{C}_{8} \mathrm{H}_{17} \mathrm{NO}_{2} \text {. Ber. C } 60.37, \mathrm{H} 1069, \mathrm{~N} 8.8 \text {. } \\
& \text { Gef. " 60.0, * 11.13, * 8.84. }
\end{aligned}
$$

Mit 10-proc. Goldchloridlösung lieferte das Betaïn in concentrirter salzsaurer Lösung ein Aurochlorat vom Schmp. 200-2020.

0.1450 g Sbst.: 0.0575 g Au. -0.1738 g Sbst.: $4.3 \mathrm{ccm} \mathrm{N}(180,749 \mathrm{~mm})$.

$\mathrm{C}_{8} \mathrm{H}_{17} \mathrm{NO}_{2} \mathrm{Cl}_{4} \mathrm{Au}$. Ber. Aa 39.95, N 2.81 .

Gef. " 39.41 , 2.81 .

$\alpha$-Triäthyl-propiobetaïn, $\left(\mathrm{C}_{2} \mathrm{H}_{5}\right)_{3} \mathrm{~N} . \mathrm{CH}\left(\mathrm{CH}_{3}\right) \cdot \mathrm{CO} . \mathrm{O}$.

Jodäthylat des Diäthylaminopropionitrils: Schmp. $178-179^{\circ}$ unter Zersetzung. Farblose Blättchen, leicht löslich in Wasser und in heissem Alkohol, unlöslich in Aether. Das Jodäthylat bildet sich in der Kälte sebr langsam, schneller beim Erbitzen in der Drackflasche auf $100^{\circ}$.

$0.1615 \mathrm{~g}$ Sbst.: $0.1358 \mathrm{~g} \mathrm{Ag} \mathrm{J}$.

$\mathrm{C}_{9} \mathrm{H}_{19} \mathrm{~N}_{2}$ J. Ber. J 45.04. Gef. J 45.44 .

1) Diese Berichte 35, 605 [1902].

7) Vergl. die Nomenclaturvorschläge Willstătter's, diese Berichte $\mathbf{3 5}$, $585[1902]$. 
Das durch Verseifung daraus erhaltene a-Triäthylpropiobetaïn schmolz, vorber erweicbend, bei $90-92^{\circ}$. Das Chloraurat krystallisirt aus heissem Wasser in kleinen Nadeln, Schmp. $253^{\circ}$.

$0.1358 \mathrm{~g}$ Sbst.: $0.3068 \mathrm{~g} \mathrm{CO}_{2}, 0.1404 \mathrm{~g} \mathrm{H}_{2} \mathrm{O} .-0.2444 \mathrm{~g}$ Sbst.: $0.554 \mathrm{~g}$ $\mathrm{CO}_{2}, 0.243 \mathrm{~g} \mathrm{H}_{2} \mathrm{O} .-0.3685 \mathrm{~g}$ Sbst: $25.4 \mathrm{ccm} \mathrm{N}\left(20^{\circ}, 766 \mathrm{~mm}\right)$.

$$
\begin{array}{llll}
\mathrm{C}_{9} \mathrm{H}_{19} \mathrm{NO}_{2} & \text { Ber. C } 62.42, & \mathrm{H} 10.98, & \text { N } 8.09 . \\
& \text { Gef. 》 } 61.6,61.75, 》 10.58,11.14, 》 7.95 .
\end{array}
$$

0.079 g Sbst.: $0.0305 \mathrm{~g} \mathrm{Au}$.

$\mathrm{C}_{9} \mathrm{H}_{20} \mathrm{NO}_{2} \mathrm{Cl}_{4} \mathrm{Au}$. Ber. Au 38.48. Gef. Au 38.35.

Methyl-diälhyl- $\alpha$-phenylbetaïn, $\left(\mathrm{CH}_{3}\right)\left(\mathrm{C}_{2} \mathrm{H}_{5}\right)_{2} \mathrm{~N} \cdot \mathrm{CH}\left(\mathrm{C}_{6} \mathrm{H}_{5}\right) \cdot \mathrm{CO} . \mathrm{O}$.

Das für die Synthese dieses Betaïns notbwendige Diäthylamino-phenylessigsäurenitril, $\left(\mathrm{C}_{2} \mathrm{H}_{5}\right)_{2} \mathrm{~N} \cdot \mathrm{CH}\left(\mathrm{C}_{6} \mathrm{H}_{5}\right) \cdot \mathrm{CN}$, wurde durch Erhitzen von Diäthylamin und Mandelsäurenitril bereitet. Es ist ein schwach gelbliches, geruchloses Oel, das bei $142^{\circ}$ unter $16 \mathrm{~mm}$ Druck unzersetzt siedet. Spec. Gewicht 0.9736 bei $19 \% 4^{0}$. $0.2067 \mathrm{~g}$ Sbst.: $0 . \dot{5} 777 \mathrm{~g} \mathrm{CO}_{2}, 0.1632 \mathrm{~g} \mathrm{H}_{2} \mathrm{O} .-0.1922 \mathrm{~g}$ Sbst.: $26.5 \mathrm{ccm}$ N $\left(25^{\circ}, 753 \mathrm{~mm}\right)$.

$$
\begin{aligned}
& \mathrm{C}_{12} \mathrm{H}_{18} \mathrm{~N}_{2} \text {. Ber. C 76.60, H } 8.51, \mathrm{~N} 14.89 \text {. } \\
& \text { Gef. 》 } 76.22 \text {, 》 8.84, " } 15.11 \text {. }
\end{aligned}
$$

Merkwürdiger Weise liess sich das Nitril weder zum Diäthylaminophenylessigester noch zur Diäthylaminophenylessigsäure verseifen ${ }^{1)}$. Bei den darauf abrielendeu Versuchen wurde entweder Diäthylamino-phenylessigsäureamid erhalten oder die Zerfallproducte der zu erwartenden Säure: Mandelsäure und Diäthylamin:

$$
\begin{aligned}
\left(\mathrm{C}_{2} \mathrm{H}_{5}\right)_{2} \mathrm{~N} \cdot \mathrm{CH}\left(\mathrm{C}_{6} \mathrm{H}_{5}\right) \cdot \mathrm{COOH}+\mathrm{H} \cdot \mathrm{OH} & =\left(\mathrm{C}_{2} \mathrm{H}_{3}\right)_{2} \mathrm{NH} \\
& +\mathrm{HO} \cdot \mathrm{CH}\left(\mathrm{C}_{6} \mathrm{H}_{6}\right) \cdot \mathrm{COOH} .
\end{aligned}
$$

Auch das Amid liess sich nicht in die Säure verwandeln. Alkoholische Salzsäure greift es wenig an; wässrige Salzsäure spaltet Diäthylamin ab. Löst man es in verdünnter Schwefelsäure und diazotirt mit Natriumnitrit, so bleibt das Amid unverändert. Siedende Pottaschelösung spaltet es in Diäthylamin, Ammoniak und Mandelsäure (Schmp.118 ). Diäthylamino-phenylessigsäureamid bildet in Alkohol leicbt lösliche, farblose Krystalle, die sich in rerdünnten Säuren unter Salzbildung lösen und durch Alkalien wieder abgeschieden werden. Es schmilat bei $143-144^{\circ}$.

1) Er. F. Sachs war so liebenswürdig, mich darauf aufmerksam zu machen, dass er analog constituirte Säureamide ebenfalls nicht verseifen konnte. Ich verweise auf seine interessanten Beobachtungen diese Berichte 35, 3325 [190:]. 
$0.2036 \mathrm{~g}$ Sbst.: $0.5222 \mathrm{~g} \mathrm{CO}_{2}, 0.1665 \mathrm{~g} \mathrm{H}_{2} \mathrm{O} .-0.1058 \mathrm{~g}$ Sbst.: $12.3 \mathrm{ccm}$ N $(200,765 \mathrm{~mm})$.

$$
\begin{aligned}
& \mathrm{C}_{12} \mathrm{H}_{18} \mathrm{~N}_{2} \mathrm{O} \text {. Ber. O } 69.90, \mathrm{H} 8.74, \mathrm{~N} 13.6 . \\
& \text { Gef. } 69.95, \otimes 9.17, 》 13.6 .
\end{aligned}
$$

Das Jodmethylat des Diäthylamino-phenylessigsäurenitrils wurde durch längeres Stehen molekularer Mengen der Substanzen in einer Kältemischung erhalten. Es bildet farblose Krystallnadelo, die bei 128-1290 schmelzen.

Methyl-diäthyl-a-phenylbetän bildet ein weisses, sehr hygroskopisches Pulver, das von $85-87^{\prime \prime}$ schmilat.

Das Golddoppelsalz fält als Oel aus, das nach einiger Zeit erstarrt.

$0.1886 \mathrm{~g}$ Sbst.: $0.4808 \mathrm{~g} \mathrm{CO}_{2}, 0.1553 \mathrm{~g} \mathrm{H}_{2} \mathrm{O} .-0.1588 \mathrm{~g}$ Sbst.: $0.5476 \mathrm{~g}$ $\mathrm{CO}_{2}, 0.1588 \mathrm{~g} \mathrm{H}_{2} \mathrm{O}$. $-0.1622 \mathrm{~g}$ Sbst.: $8.7 \mathrm{~cm} \mathrm{~N}\left(18^{0}, 747 \mathrm{~mm}\right.$ ).

$$
\begin{aligned}
& \mathrm{C}_{13} \mathrm{H}_{19} \mathrm{NO}_{2} \text {. Ber. C 70.6, H 8.6, N 6.33. } \\
& \text { Gef. » 69.5, 70.8, 》 9.2, 8.4, »6.08. }
\end{aligned}
$$

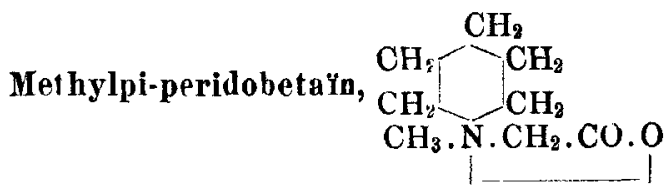

Piperido-acetonitril') wurde durch Erhitzen molekularer Mengen Glykolsäurenitril und Piperidin gewounen. Es ist eine fast gernchlose, unter $15 \mathrm{~mm}$ Druck ron $99-100^{\circ}$ anzersetzt siedende Base, die leicht zu derben, glänzenden Krystallen erstarrt. Schmp. 190. Spec. Gewicht $0.967814^{\circ} / 4^{0}$.

$0.1631 \mathrm{~g}$ Sbst. $30.6 \mathrm{ccm} \mathrm{N}\left(12^{0}, 768 \mathrm{~mm}\right)$.

$\mathrm{C}_{7} \mathrm{H}_{12} \mathrm{~N}_{2}$. Ber. N 22.58. Gef. N 22.51.

Die Base vereinigt sich mit Jodmethyl bei gewöhnlicher Temperatur leicht zu dem Jodmetbylate des Piperido-acetonitrils, das farblose Blättchen vom Schmp. 192-1930 bildet.

$0.1077 \mathrm{~g}$ Sbst.: $0.0458 \mathrm{~g} \mathrm{AgJ}$.

$$
\mathrm{C}_{3} \mathrm{H}_{15} \mathrm{~N}_{2} J \text {. Ber. J 47.74. Gef. J } 48.06 \text {. }
$$

Mit Silberoxyd lieferte das Jodmethylat leicht die entsprechende quartäre Base, aus der durch Kochen mit Barythydrat Methylpiperidobetaïn gewonn $n$ wurde.

Methylpiperidobetaïn bildet ein farbloses, hygroskopisches Pulver, das von $116-118^{\prime}$ unter vorhergehendem Erweichen schmilzt.

1) Piperidoacetonitril ist nach einer Privatmittheilung bereits von Henry dargestellt worden. 
$0.1855 \mathrm{~g}$ Sbst.: $0.4170 \mathrm{~g} \mathrm{CO}_{2}, 0.1543 \mathrm{~g} \mathrm{H}_{2} \mathrm{O} .-0.2082 \mathrm{~g}$ Sbst.: $16.9 \mathrm{~cm}$ N $\left(18^{\circ}, 745 \mathrm{~mm}\right)$.

$$
\begin{array}{ll}
\mathrm{C}_{8} \mathrm{H}_{15} \mathrm{NO}_{2} . & \text { Ber. } \mathrm{C} 61.15, \mathrm{H} \mathrm{9.55,} \mathrm{N} \mathrm{8.9.} \\
& \text { Gef. } \$ 61.39, * 9.55, » 8.8 .
\end{array}
$$

Das gold cblorwasserst of f a ure Salz krystallisirte aus Wasser in kleinen Nadeln. Schmp. 178-1799.

$0.1248 \mathrm{~g}$ Sbst.: $0.0498 \mathrm{~g} \mathrm{Au}$.

$\mathrm{C}_{8} \mathrm{~B}_{15} \mathrm{NO}_{2} \mathrm{Cl}_{3} \mathrm{Au}$. Ber. Au 39.71. Gef. Au 39.90 .

Ich beabsichtige, die quartären Nitrile elektrolytisch zu reduciren und die Synthese von Cholinen und Neurinen auf diese Weise durchzuführen.

Heidelberg, Universitätslaboratorium.

\section{M. Iljinsk y: Darstellung isomerer Sulfosäuren mittels Katalyse.}

[Mitth. ans dem Laborat. der Alizarinfabrik von R. Wedekind \& C•., Uerdingen a. Rhein.]

(Eivgegangen am 25. November 1903.)

Die Einfübrung der Sulfogruppen in die organischen Verbindungen mittels directer Einwirkung von Schwefelsäure erfolgte bis jetzt in jedem gegebenen Falle nur in ganz bestimmten Stellungen. So war es bis jetzt z. B. unmöglich, durch directes Sulfiren von Anthrachinon zu anderen als in der $m$-Stellung substituirten Derivaten zu gelangen. Gnehm ${ }^{1}$ ) scbreibt darüber: $\$$ Wird Anthrachinon mit Schwefelsäure sulfirt, so treten die $\mathrm{SO}_{3} \mathrm{H}$-Gruppen stets in Metastellung und nie in Ortbostellung zu einer CO-Gruppe. Werden zwei $\mathrm{SO}_{3} \mathrm{H}$-Gruppen eingeführt, so vertheilen sie sich auf die beiden Kerne. Es können demnach die drei einzig möglichen Säuren, eine Monosulfosäure und zwei Disulfosäuren von folgender Constitution entstehen:

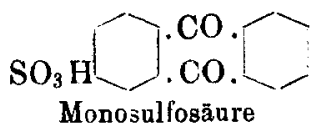<smiles>O=C(O)C1C(=O)CCC[C@H]1OS(=O)(=O)O</smiles>
a Disulfosäure

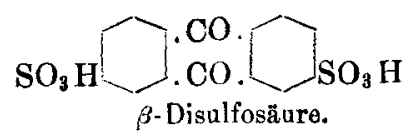

Ortbosulfosãuren bilden sich nur durch Oxydation der Anthracensulfosăuren.»

1) Die Anthracenfarbstoffe (1897), S. 19. 